\title{
FABRICATION AND ELECTRICAL CHARACTERIZATION OF MICROSCALE DIELECTRIC BARRIER DISCHARGE DEVICES
}

\author{
Justin C. Zito*, and David P. Arnold \\ Interdisciplinary Microsystems Group, University of Florida, Gainesville, Florida, USA
}

\begin{abstract}
In this paper we report the microfabrication and electrical characterization of microscale dielectric barrier discharge (DBD) plasma devices on glass substrates. The devices employ $\mathrm{Au}$ electrodes $(5 \mathrm{~mm}$ long, $\leq 40 \mu \mathrm{m}$ wide) separated by a polyimide dielectric (10 $\mu \mathrm{m}$ or $50 \mu \mathrm{m}$ thick). Under ac sinusoidal excitation at $14 \mathrm{kHz}$, the plasma discharges ignite around $1 \mathrm{kV}_{\mathrm{pk}}$ input amplitude. The power consumption of a single device is on the order of $3-6 \mathrm{~mW} / \mathrm{mm}$ and power delivery is found to increase linearly when multiple devices are connected in parallel. The effective plasma load impedance is extracted from the measured voltage/current waveforms, and the parameters for a parallel $\mathrm{R}-\mathrm{C}$ load model are computed. Since most of the tested devices sustained plasma for only a few minutes before failure, reliability of the devices is also briefly discussed.
\end{abstract}

\section{INTRODUCTION}

\section{Background \& Motivation}

Atmospheric plasma discharge devices are finding growing application in aerodynamic flow control, biological sterilization, water purification (ozone generation), surface treatment (e.g. corona charging), and more recently microfluidic propulsion [1]. For flow control and propulsion applications, advantages of plasma actuators include a lack of moving parts, construction simplicity, and near instantaneous response.

As shown in Figure 1, a standard DBD device consists of two parallel electrodes offset from one another on either side of a dielectric material. The top electrode is exposed to air while the bottom electrode is encapsulated by another dielectric to prevent discharge on the bottom side of the device. A high-voltage dc or ac source is applied to the exposed (top) electrode, while the lower electrode is grounded. When a sufficiently high voltage is applied to the electrodes, the air weakly ionizes near the surface of the dielectric. This imparts an electrohydrodynamic (EHD) body force to the fluid, providing momentum to the flow.

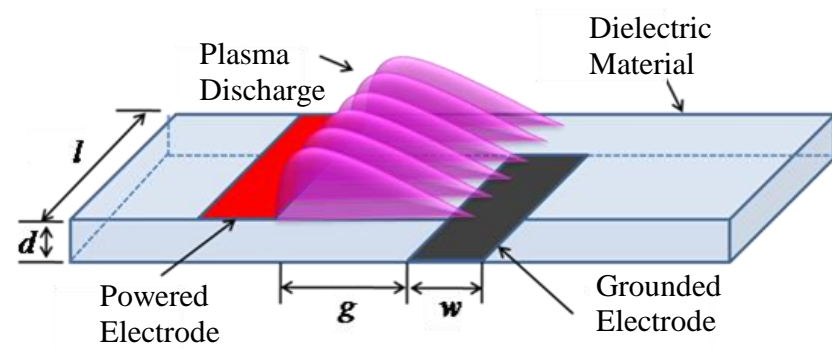

Figure 1: Schematic of a single dielectric barrier discharge (DBD) plasma device.

One motivation for exploring microscale DBD devices stems from numerical simulations of parallel plate volume discharges that suggest a significant increase in the electric force density $\left(\mathrm{N} / \mathrm{m}^{3}\right)$ may be achievable using micron-sized electrode gaps [2]. The numerical predictions of [2] showed close agreement with electric field measurements from micron-scale gapped devices [3], thus building confidence in the numerical models. While difficult to experimentally measure, the charge density and electric force were computed using the numerical models. For electrode gaps below $\sim 30 \mu \mathrm{m}$, the electric force density is estimated to increase by over two orders of magnitude. While these simulations and experiments were for parallel-plate volume discharges, if higher force densities can similarly be achieved for surface-discharge DBD configurations, this could have significant impact on flow control or propulsion devices.

A brief explanation for the increased force density is as follows. With decreasing gap, the sheath region of the plasma discharge occupies a greater portion of the discharge, reducing the quasi-neutral region (where electron density $\simeq$ ion density) [2]. The momentum transfer to the fluid is due to the Lorentz force, $q E$, where $q$ is the net charge separation, and $E$ is the electric field. Since the sheath region has the largest charge separation $q$, (the ion density is much higher than the electron density in the sheath region), using a smaller gap should provide a larger electric force density, and thus increasing the local body force to the fluid.

Further motivation for microscale discharge is that the voltage required to achieve the electric field necessary for plasma ignition is reduced. This lowers the power requirements for device operation, thus translating to smaller and lighter power supplies for flight vehicles. Moreover, batch microfabrication enables highthroughput manufacturing of complex electrode shapes with good alignment and dimensional control. The ability to fabricate electrodes on flexible substrates is also of great interest.

\section{Prior Work}

In addition to the work of Longwitz [3], other researchers have begun to investigate microscale discharge devices. Ono et al. [4] investigated the breakdown threshold voltages for parallel-plate micro-gaps on the order from $2-50 \mu \mathrm{m}$. They showed that breakdown voltages for Si-Si electrodes closely follows Paschen's curve, though Si-metal breakdown voltages deviated significantly from Paschen's curve at gap widths below $5 \mu \mathrm{m}$. Ono et al. [5] also showed that both metal and $\mathrm{Si}$ electrodes are subject to sputtering/erosion during the discharge process. The evaporated material increases the local pressure in the gap region, resulting in a decrease in the breakdown threshold voltage. This erosion is an important consideration in the reliability of micro discharge devices, since this may limit the time before failure. Bass et al. [6] developed a capacitive-coupled helium microplasma that was formed in a narrow channel by electrodes on either side of a quartz plate. This design was intended as an atomic emission detector having a minimum channel cross-section of $0.2 \times 0.2 \mathrm{~mm}$, and can be thought of as a DBD volume discharge device. Mitra and Gianchandani [7] developed pulsed micro-discharge devices using two- and three-electrode geometries with a $200 \mu \mathrm{m}$ electrode gap for use in species detection and optical emission spectroscopy. Okochi et al. [8] have developed and tested mm-size DBD plasma devices using MEMS fabrication processes.

\section{This Work}

In this paper, we focus our efforts on generating DBD surface plasma using electrodes and gaps with microscale dimensions. As shown in Figure 2, the electrode widths are $20 \mu \mathrm{m}$ (top) and $40 \mu \mathrm{m}$ (bottom), and each electrode is $5 \mathrm{~mm}$ long (spanwise). The lateral gap between the electrodes ranges from $50-120 \mu \mathrm{m}$. The 
electrodes are $300 \mathrm{~nm}$ thick ( $50 \mathrm{~nm} \mathrm{Ti}, 250 \mathrm{~nm} \mathrm{Au}$ ), separated by a polyimide dielectric, of either $10 \mu \mathrm{m}$ or $50 \mu \mathrm{m}$. The structures are fabricated on soda lime glass substrates. Polyimide was chosen for its good metal adhesion and its common use as a dielectric in macroscale DBD plasma devices. Rounded edges are used to avoid field-concentration points and resulting plasma "streamers."
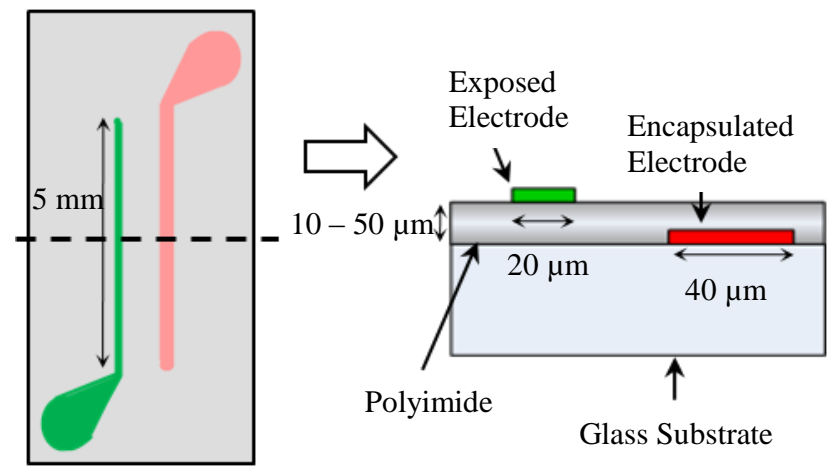

Figure 2. Top view and cross-section schematics of DBD devices.

\section{DEVICE FABRICATION}

The process flow for these devices is shown in Figure 3. The first steps involve sputtering and lift-off to define the lower Ti/Au electrodes. The Ti layer is used to improve the adhesion of the Au. A low-stress polyimide (HD Microsystems PI2611) is used as the 'barrier' or insulation layer. The polyimide was spun and softbaked multiple times to reach the desired dielectric thickness. The substrate is then placed on a hotplate to cure the polyimide layer. Before patterning the top electrodes, the polyimide is etched with an $\mathrm{O}_{2}$-based reactive ion etch (RIE) to open contacts to the lower electrode bond pads (not shown in the cross-sections). A sputtered chrome layer was used as the etch mask for the RIE process. Following the polyimide etch, the chrome mask is removed using a wet chrome etchant. The top electrodes are then sputtered and patterned using liftoff.
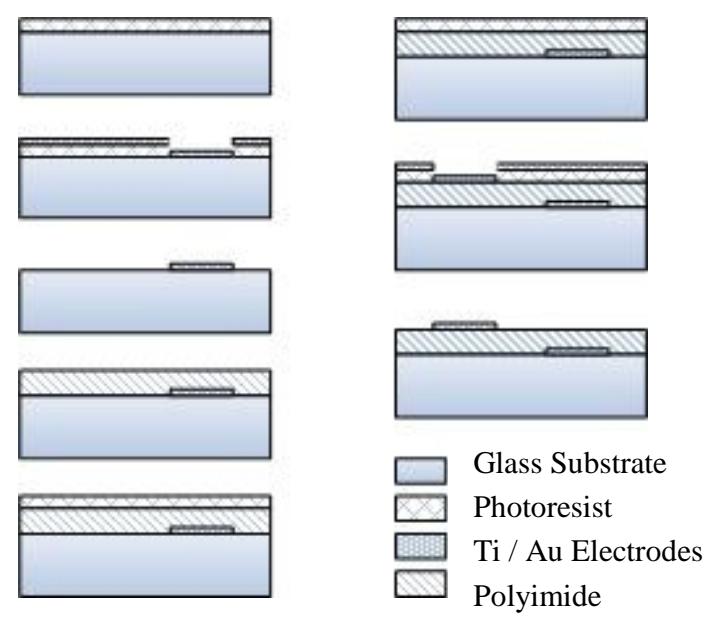

Figure 3. Fabrication process for DBD plasma devices.

\section{EXPERIMENT}

Optical images of various completed devices are shown in Figure 4. Directed plasma discharge is shown (left-to-right for the linear designs, top-to-bottom for the curved design) across the micro-gap in three of the four images. While various electrode configurations were fabricated, the remainder of this paper focuses on test results from the simple straight-electrode configuration, both single devices and parallel connected devices. The parallel connected designs comprised 4 to 6 devices connected together, which all have the same gap.

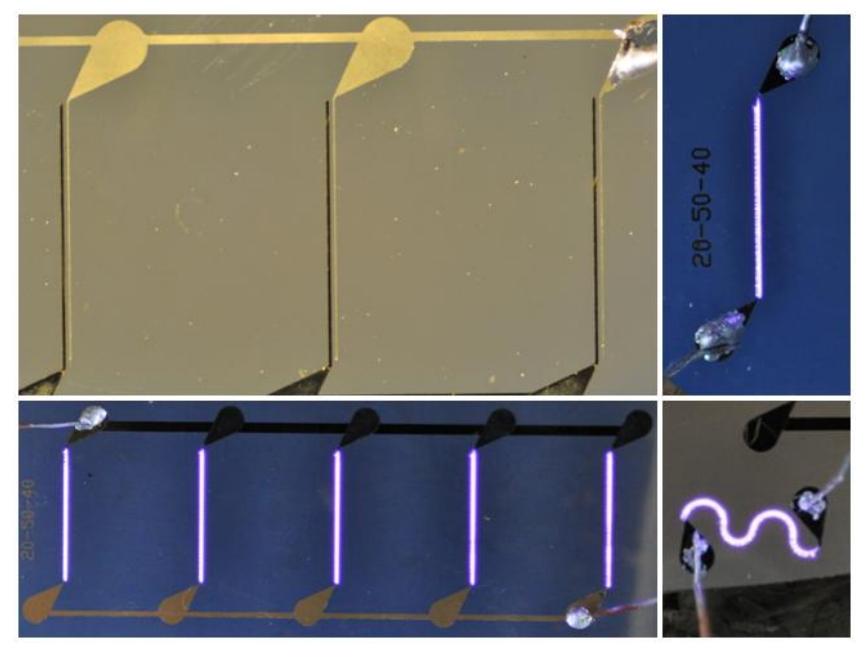

Figure 4. Various DBD plasma devices; clockwise from top right: single actuator with $50 \mu \mathrm{m}$ gap; serpentine geometry, $g=80 \mu \mathrm{m}$; five actuators in parallel, $g=50 \mu \mathrm{m}$; fabricated parallel devices (no discharge present) showing electrode detail.

\section{Test Set-Up}

The schematic for the experiment set-up is shown in Figure 5. The devices were tested using ac sinusoidal excitation at $14 \mathrm{kHz}$, with input amplitude on the order of $1 \mathrm{kV}_{\mathrm{pk}}$. The power supply consisted of a standard function generator (Tektronix AFG 3022B), an audio amplifier (QSC Audio RMX 2450) to increase the signal to the 10's of volts range, and a custom transformer (Corona Magnetics, Inc.) to further increase the voltage into the $\mathrm{kV}$ range. A digital oscilloscope (Tektronix DPO 3014) was used to capture the voltage and current waveforms. A Tektronix P6015A high-voltage probe was used, along a Pearson 2100 current probe. The current probe has a $20 \mathrm{MHz}$ bandwidth, and the oscilloscope was set with a $20 \mathrm{MHz}$ bandwidth limit for the current channel.

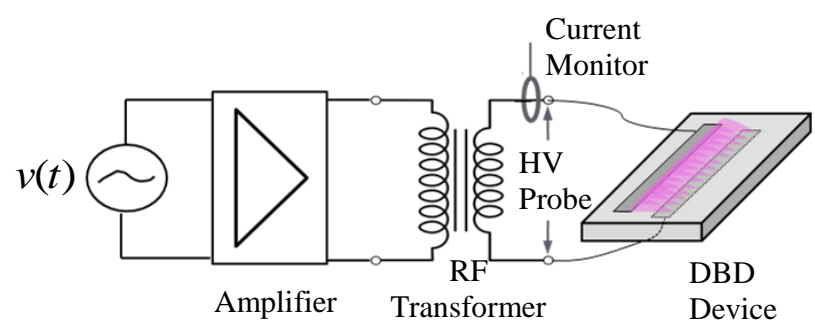

Figure 5. Schematic of the experiment set-up.

For each device tested, 10 data records were captured. Each data record consisted of one million data points, sampled at 250 $\mathrm{MS} / \mathrm{s}$, corresponding to 56 periods of data within each record. The 10 data records yielded a total of 560 periods of data for analysis. The data was analyzed to determine average power consumption and equivalent impedance characteristics.

Ideally, several different devices were tested for each gap width and dielectric thickness. Due to burn-out and reliability issues (discussed in a later section), the number of devices that produced stable discharge that allowed for data collection was not consistent for each geometry. Hence, the number of devices tested for each geometry varied from 2 to 10 . 


\section{Parameter Extraction}

The average power is calculated by integration of the product of the voltage and current waveforms. Discretely, this was done by the point-by-point multiplication of the voltage and current waveforms, which is then summed and divided by the number of data points. From the voltage and current waveforms, load impedance parameters can be extracted, assuming a simple $\mathrm{R}-\mathrm{C}$ circuit model to represent the impedance behavior of the plasma. The extraction process follows a previously developed plasma impedance extraction methodology, described in [9]. The resistance is used to model the real power consumed by the device, while the capacitance is used to represent the reactive behavior.

\section{RESULTS}

Plasma was typically ignited around $1 \mathrm{kV}_{\mathrm{pk}}$ input amplitude, much lower than the $5+\mathrm{kV}_{\mathrm{pk}}$ range required for macroscale $\mathrm{DBD}$ devices. The average power consumed by a single device is plotted against varying gap widths in Figure 6. The voltage necessary to create discharge for the devices with a $50 \mu \mathrm{m}$ dielectric is, on average, $140 \mathrm{~V}$ higher in amplitude than that for the $10 \mu \mathrm{m}$ dielectric devices, although the average power consumed is greater for the thinner dielectric. This may be attributed to a better conduction path established with the $10 \mu \mathrm{m}$ barrier, causing more current flow and increased power consumption. The data shows a small increase in the average power consumed with increasing gap size, most likely due to an increase in the input voltage required to generate discharge with larger gap sizes. (The device with $110 \mu \mathrm{m}$ gap did not create a discharge that remained stable for data collection. It would fail during the data capture process and did not provide reliable data.) The uncertainties (error bars) shown in the figure were computed using a t-distribution with a $95 \%$ confidence interval. For some of the gap sizes, only a few devices were successfully tested and hence the corresponding uncertainty is significant.

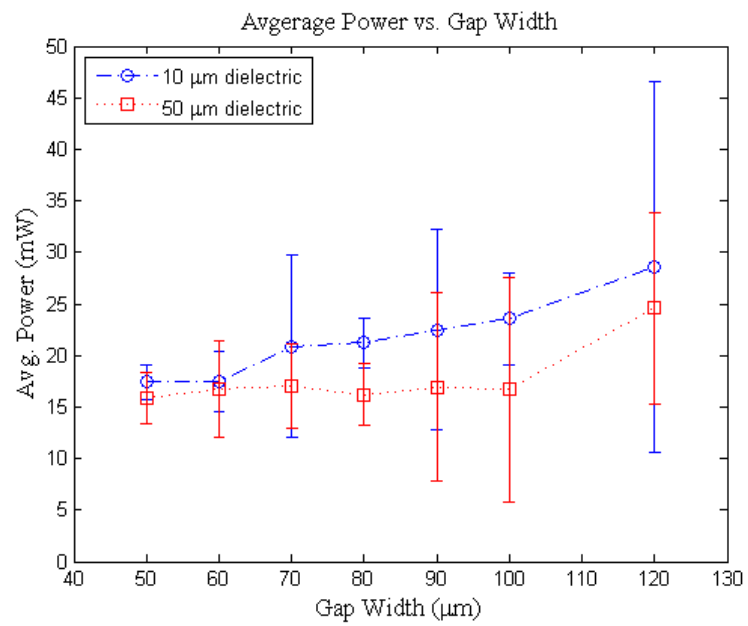

Figure 6. Average power consumed by a single device plotted against varying gap width.

Figure 7 compares the average power consumption with the number of devices connected in parallel for devices with a $10 \mu \mathrm{m}$ thick dielectric. The data implies that an increased amount of power is necessary to actuate several plasma devices with an apparently linear trend, though more data is required to better quantify the relationship between the power requirement and number of devices.

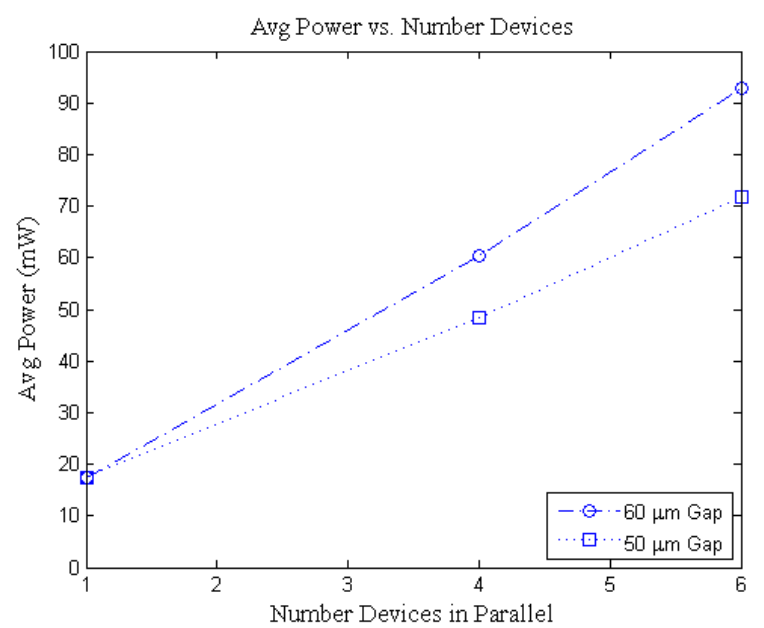

Figure 7. Average power consumed plotted against number of devices connected in parallel. Data shown for linear electrodes with $g=50 \mu \mathrm{m}$ and $60 \mu \mathrm{m}$, and a $10 \mu \mathrm{m}$ thick dielectric.

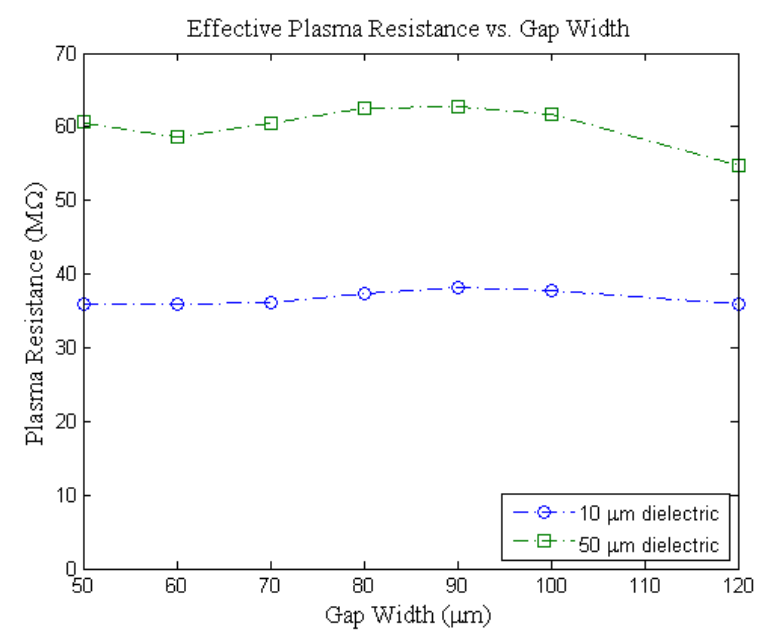

Figure 8. Effective plasma load resistance plotted against varying gap width.

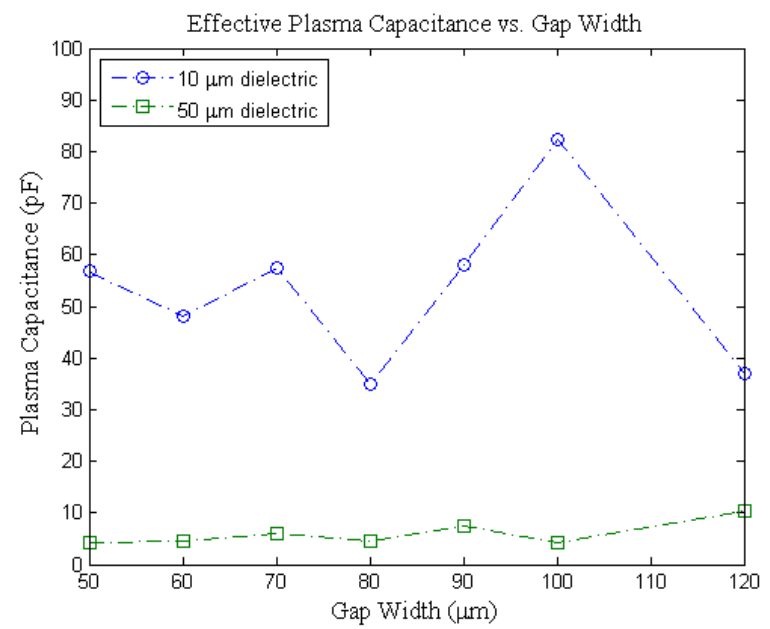

Figure 9. Effective plasma load capacitance plotted against varying gap width. 
The effective plasma load resistance and capacitance were calculated and are plotted in Figures 8 and 9, respectively. Both the resistance and capacitance values remain fairly constant as a function of gap $(\mathrm{g})$. The resistance values fluctuate by up to $5 \%$ of their mean value. The capacitance values vary significantly more, by about $35 \%$ of its mean value. There is a clear difference between the two dielectric thicknesses, however. The thinner dielectric barrier has less resistance and higher capacitance. The larger capacitance values are consistent with a smaller dielectric thickness. Compared to macroscale DBD devices [9], these microscale devices exhibit smaller resistance and larger capacitance, also consistent with the smaller physical dimensions.

\section{Reliability Concerns}

While prior work by the researches have shown quite reliable and stable DBD plasma devices at the macroscale, these microscale DBD devices seem more prone to failure. Electric field concentration points often result in an intense "streamer" of plasma which erodes the dielectric barrier (as opposed to a uniform glow discharge). The length of time for which discharge was sustained varied among the devices tested. A device was considered "successful" if it sustained discharge for over one minute. A few devices sustained discharge for upwards of five minutes without failure. At the other extreme, some devices would fail before generating a stable discharge.

The source of failure may come from a combination of variables, such as non-uniformities in the dielectric material, jagged electrode edges, and/or electrode erosion eventually leading to failure. The quality, or smoothness, of the electrodes is thought to be a significant factor in the device reliability. Fabrication details such as photomask quality, metal deposition and metal patterning process may all have an impact on device performance and reliability. The three images in the left half of Figure 10 show examples of several failed devices, showing that points of failure most often occur near one of the ends of the powered electrode. Areas of charred/burned dielectric can be identified near the ends of the electrodes in the three images on the left. The three images in the right half of Figure 10 show example devices just after/at the onset of failure.

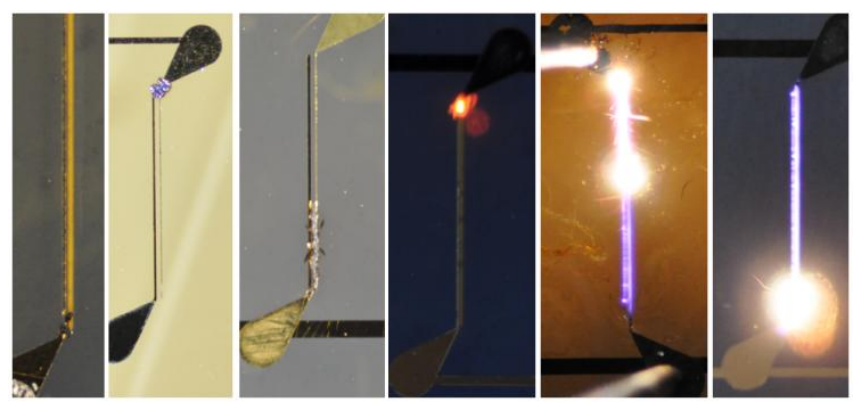

Figure 10. Images of device failure; the left three images show devices after failure; the right three images show devices at the onset of failure.

\section{CONCLUSION}

Microscale DBD plasma devices were successfully fabricated and demonstrated sustainable plasma discharge. The power requirement for these devices depends upon the number and length of the devices used, although for a limited number of devices the power requirement was between $3-6 \mathrm{~mW} / \mathrm{mm}$ for a single device. The power requirement appears to scale linearly with increasing number of devices, although more data are necessary to make a definite conclusion. The impedance data are similar, qualitatively, to the trends reported for macroscale DBD devices [9]: the effective plasma resistance is lower for the thinner $(10 \mu \mathrm{m})$ dielectric, while the effective capacitance and the average power consumed are greater for the thinner $(10 \mu \mathrm{m})$ dielectric.

The reliability of DBD devices remains an open topic for exploration. As discussed previously, local points of concentrated electric field often lead to device failure and are difficult to mitigate. The source of failure may come from a combination of variables, from the material properties/defects to the fabrication processes used. Electrode erosion is an inevitable process that is a byproduct of micro-discharge devices and must be further studied.

Ongoing efforts aim to characterize the flow actuation of these devices. The ultimate goal is to improve the performance of these devices by imparting the maximum momentum to the flow. To characterize their flow effects, micro particle imaging velocimetry (micro-PIV) can be used to collect velocity measurements. Other polymer materials, such as SU-8, PMMA, PDMS, and KMPR, as well as thinner inorganic dielectrics are also being investigated for use as the dielectric barrier layer. Furthermore, reductions of the gap width and dielectric thickness are to be fabricated and tested as well.

\section{ACKNOWLEDGEMENTS}

This work was supported in part by the US Air Force Office of Scientific Research (AFOSR) grant \#FA9550-09-1-0416.

\section{REFERENCES}

[1] E. Moreau, "Airflow Control by Non-Thermal Plasma Actuators," J. Phys. D: Appl. Phys., 40 (2007), pp. 605-636.

[2] C.-C. Wang, and S. Roy, "Microscale Plasma Actuators for Improved Thrust Density", J. Appl. Phys., 106, (2009).

[3] R.G. Longwitz, "Study of Gas Ionization in a Glow Discharge and Development of a Micro Gas Ionizer for Gas Detection and Analysis", Ph.D. Thesis, Swiss Federal Institute of Technology, Lausanne, Switzerland, 2004.

[4] T. Ono, D.Y. Sim, and M. Esashi, "Micro-Discharge and Electric Breakdown in a Micro-Gap", J. Micromech. Microeng., 10 (2000), pp. 445 - 451.

[5] T. Ono, D.Y. Sim, and M. Esashi, "Imaging of MicroDischarge in a Micro-Gap of Electrostatic Actuator", MEMS $2000,13^{\text {th }}$ Annual Int'l Conf. on, Miyazaki, Japan (2000), pp. $651-656$.

[6] A. Bass, C. Chevalier, and M.W. Blades, "A Capacitively Coupled Microplasma $(\mathrm{CC} \mu \mathrm{P})$ Formed in a Channel in a Quartz Wafer", J. Anal. At. Spectrometry, 16 (2001), pp. 919 $-921$.

[7] B. Mitra, and Y.B. Gianchandani, "The Detection of Chemical Vapors in Air Using Optical Emission Spectroscopy of Pulsed Microdischarges From Two- and Three- Electrode Microstructures," J. IEEE Sensors, 8, 8 (2008), pp. 1445-1454.

[8] S. Okochi, N. Kasagi, Y. Suzuki et al., "Development of Micro Plasma Actuator for Active Flow Control", Exp. Heat Transfer, Fluid Mechanics and Thermodynamics, $7^{\text {th }}$ World Conf. on, Krakow, Poland (2009).

[9] J. Zito, R. Durscher, D.P. Arnold et al., "Investigation of Impedance Characteristics and Power Delivery for Dielectric Barrier Discharge Plasma Actuators", Proceedings, $48^{\text {th }}$ AIAA Aerospace Sciences Meeting, Orlando, FL (2010).

\section{CONTACT}

*J.C. Zito, tel: +1-352-392-1484; jcz@ufl.edu 